\title{
Induction Motor Stator Fault Detection by a Condition Monitoring Scheme Based on Parameter Estimation Algorithms
}

\author{
FANG DUAN*
}

\section{RASTKO ŽIVANOVIĆ ${ }^{\dagger}$}

\author{
*School of Engineering \\ London South Bank University, London, UK \\ ${ }^{\dagger}$ School of Electrical and Electronic Engineering \\ The University of Adelaide, Adelaide, SA, Australia
}

\begin{abstract}
This paper presents a simple, low-cost and effective method for the early diagnosis of stator short circuit faults. The approach relies on the combination of an induction motor mathematical model and parameter estimation algorithm. Kernel of the method is the efficient search for the characteristic parameters that are indicating stator short circuit faults. However, the nonlinearity of a machine model may imply multiple local minima of an objective function implemented in the estimation algorithm. Taking this into consideration, we investigate suitability of two industry-proven optimization algorithms (pattern search algorithm and genetic algorithm) as applied in the proposed condition monitoring method. Experimental results show that the proposed diagnosis method is capable of detecting stator short circuit faults and estimating level and location of faults. The study also indicates that the proposed method is robust to motor parameters offset and unbalanced voltage supply. Application of the pattern search algorithm is suitable for a continuous monitoring system, where the previous result can be used as starting point of the new search. The genetic algorithm requires longer computation time and it is suitable for the offline diagnostic system. It is not sensitive to starting point and achieving global solution is guaranteed.
\end{abstract}

Manuscript received in final form on May 14, 2014.

Address correspondence to Dr Fang Duan, School of Engineering, London South Bank University, 103 Borough Road, London, UK, SE1 0AA. E-mail: duanf@lsbu.ac.uk 


\section{Introduction}

Induction motors are the most widespread rotating electric machines in industry due to their efficient and cost-effective performance. An induction motor failure results in severe damage not only to the motor itself but also to motor-related equipment devices in an industrial plant. Consequently, motor condition monitoring is of great necessity to detect motor faults at the early stage in order to reduce unscheduled downtime, repair costs, and increase life span of machines.

Condition monitoring is the process of monitoring parameters that are describing condition of a machine while in operation. Through the parameters monitoring process, motor failures can be diagnosed before irreversible damage has occurred. The condition monitoring can be broadly categorized into two basic types: intrusive and non-intrusive techniques [1]. The intrusive method is complex and costly because particular sensors and measurement equipments have to be employed to monitor the deviation of air-gap torque [2], magnetic field [3], vibration [4] and so on. For the non-intrusive method, the condition monitoring can be achieved by processing of supply voltage and current signals. Therefore, the advantages of the non-intrusive method are reduced cost and simplicity.

Motor current signature analysis (MCSA) is one of the most widely applied non-intrusive methods. Motor operating conditions can be obtained by monitoring stator current [5]. Induction motor faults are diagnosed by monitoring a variety of fault signatures extracted from the current signal, such as current Park's vector [6], current-envelope analysis [7], phase-shift analysis [8], residual saturation harmonics [9], current residue decomposition [10], and the symmetrical component analysis [11]. These methods are conducted in either time-domain or frequency- 
domain. Frequency-domain analysis can provide more detailed information of machine's status and Fast Fourier Transform (FFT) spectral signature analysis is a commonly used method $[9,11]$. However, the harmonic frequency components are too small to be detected under the low intensity fault condition, which limits application of those methods [12]. Alternative techniques such as Zoom FFT [13], discrete wavelet transformation [14], Teager-Kaiser Energy Operator [15] and improved Hilbert method [16] have been proposed to enhance frequency resolution and improve the performance.

The induction motors are symmetrical structures and any kind of fault will break their symmetrical property. The change of this balance will result in the drift of characteristic parameters, which offers a method to detect motor fault by monitoring these parameters [12]. For example, the stator short circuit fault has been successfully detected through the estimation of characteristic parameters from the recorded stator current [17]. The parameter estimation technique is based on a MATLAB/SIMULINK induction motor model and a global direct search algorithm, named Hyperbolic Cross Points (HCP) algorithm [18]. It is worthwhile to mention that global search algorithm has to be employed since the local search methods are very sensitive to the selection of an initial point. The local search method might be trapped into local minima if its start point is far from global minimum.

In this paper, the industry-proven optimization algorithms: pattern search algorithm (PSA) [19] and genetic algorithm (GA) [20] are employed to monitor motor condition. Two parameters (localization parameter and fault level) are able to characterize fault conditions of the stator winding and fault diagnostic systems can be based on the estimation of those parameters [21]. Estimation is computed by using various algorithms for searching global minimum of the objective function. As a di- 
rect local search algorithm, the PSA is sensitive to starting (i.e. initial) point. The algorithm might be trapped into local minima. To this end, the Latin hypercube sampling (LHS) method [22] is utilized to generate a proper starting point for the PSA. On the contrary, the GA is one of the artificial intelligence approaches and it is based on the Darwin theory of evolution [20]. Although GA requires longer computation time to estimate parameters, it guarantees detection of a global minimum. We applied these two optimization algorithms to estimate parameters describing various stator fault conditions of an induction motor under different loading levels. The faults diagnosis also considers the offset of motor parameters and unbalanced voltage supply. Practical results obtained in the laboratory environment by using both PSA and GA algorithms for the stator fault diagnostic task are analyzed and compared in terms of accuracy and computational cost.

\section{Mathematical model of a stator short circuit fault in an induc- tion motor}

The stator short circuit fault in an induction motor is normally characterized by two parameters: $\eta_{f}$ fault level and $\theta_{f}$ fault location. The fault localization $\theta_{f}$ of $\{0,1,2\} \times 2 \pi / 3$ stands for Phase $\{a, b, c\}$, respectively. An induction motor model with stator short circuit fault has been previously built based on a mathematical model in [21,23]. The proposed model has been validated by comparing simulated and measured stator currents with different number of shorted turns and different load levels. In this paper, the model has been adapted and utilized in the parameter estimation algorithm. For the sake of convenience, the mathematical model of induction motor with short circuit fault is briefly described in matrix form as follows: 


$$
\begin{gathered}
\frac{\mathrm{d}}{\mathrm{d} t}\left[\begin{array}{c}
\boldsymbol{\lambda}_{s}^{a b c} \\
\boldsymbol{\lambda}_{r}^{a b c} \\
\lambda_{f}
\end{array}\right]=\left[\begin{array}{c}
\boldsymbol{u}_{s}^{a b c} \\
0 \\
0
\end{array}\right]-\boldsymbol{r}\left[\begin{array}{c}
\boldsymbol{i}_{s}^{a b c} \\
\boldsymbol{i}_{r}^{a b c} \\
i_{f}
\end{array}\right], \\
{\left[\begin{array}{c}
\boldsymbol{\lambda}_{s}^{a b c} \\
\boldsymbol{\lambda}_{r}^{a b c} \\
\lambda_{f}
\end{array}\right]=\left[\begin{array}{lll}
\boldsymbol{L}_{s s}^{a b c} & \boldsymbol{L}_{s r}^{a b c} & \boldsymbol{L}_{s f}^{a b c} \\
\boldsymbol{L}_{r s}^{a b c} & \boldsymbol{L}_{r r}^{a b c} & \boldsymbol{L}_{r f}^{a b c} \\
\boldsymbol{L}_{f s} & \boldsymbol{L}_{f r} & L_{f}
\end{array}\right]\left[\begin{array}{c}
\boldsymbol{i}_{s}^{a b c} \\
\boldsymbol{i}_{r}^{a b c} \\
i_{f}
\end{array}\right],}
\end{gathered}
$$

where

$\boldsymbol{\lambda}_{s}^{a b c}, \mathbf{u}_{s}^{a b c}$ and $\mathbf{i}_{s}^{a b c}$ stator flux, voltage and current

$\boldsymbol{\lambda}_{r}^{a b c}, \mathbf{u}_{r}^{a b c}$ and $\mathbf{i}_{r}^{a b c}$ rotor flux, voltage and current

$\lambda_{f}$ and $i_{f} \quad$ short circuit flux and current

$\mathbf{r}=\left[\begin{array}{lll}\boldsymbol{r}_{s} & 0 & 0 \\ 0 & \boldsymbol{r}_{r} & 0 \\ 0 & 0 & r_{f}\end{array}\right]$

$\mathbf{r}_{s}=r_{s} \times \boldsymbol{I}$

stator resistance

$\mathbf{r}_{r}=r_{r} \times \boldsymbol{I}$

rotor resistance

$r_{f}=\eta_{f} \cdot r_{s}$

short circuit resistance

$\boldsymbol{I}=\left[\begin{array}{lll}1 & 0 & 0 \\ 0 & 1 & 0 \\ 0 & 0 & 1\end{array}\right]$

Identity matrix

$\boldsymbol{L}_{s s}=\left[\begin{array}{ccc}L_{g}+L_{p} & -L_{p} / 2 & -L_{p} / 2 \\ -L_{p} / 2 & L_{g}+L_{p} & -L_{p} / 2 \\ -L_{p} / 2 & -L_{p} / 2 & L_{g}+L_{p}\end{array}\right]$, 


$$
\begin{gathered}
\boldsymbol{L}_{r r}=\left[\begin{array}{ccc}
L_{p} & -L_{p} / 2 & -L_{p} / 2 \\
-L_{p} / 2 & L_{p} & -L_{p} / 2 \\
-L_{p} / 2 & -L_{p} / 2 & L_{p}
\end{array}\right], \\
L_{f}=\eta_{f}^{2}\left(L_{p}+L_{g}\right)
\end{gathered}
$$

where $L_{p}$ and $L_{g}$ are mutual inductance and self inductance referred to the stator, respectively.

The stator, rotor and short-circuit mutual inductances can be expressed as [21],

$$
\begin{gathered}
\boldsymbol{L}_{s r}=L_{p}\left[\begin{array}{ccc}
\cos \left(\theta_{r}\right) & \cos \left(\theta_{r}+2 \pi / 3\right) & \cos \left(\theta_{r}-2 \pi / 3\right) \\
\cos \left(\theta_{r}-2 \pi / 3\right) & \cos \left(\theta_{r}\right) & \cos \left(\theta_{r}+2 \pi / 3\right) \\
\cos \left(\theta_{r}+2 \pi / 3\right) & \cos \left(\theta_{r}-2 \pi / 3\right) & \cos \left(\theta_{r}\right)
\end{array}\right], \\
\boldsymbol{L}_{s f}=\eta_{f} L_{p}\left[\begin{array}{c}
\cos \left(\theta_{f}\right) \\
\cos \left(\theta_{f}-2 \pi / 3\right) \\
\cos \left(\theta_{f}+2 \pi / 3\right)
\end{array}\right], \\
\boldsymbol{L}_{r f}=\eta_{f} L_{p}\left[\begin{array}{c}
\cos \left(\theta_{f}-\theta_{r}-2 \pi / 3\right) \\
\cos \left(\theta_{f}-\theta_{r}+2 \pi / 3\right)
\end{array}\right],
\end{gathered}
$$




$$
\boldsymbol{L}_{r s}=\boldsymbol{L}_{s r}^{T}, \quad \boldsymbol{L}_{f s}=\boldsymbol{L}_{s f}^{T}, \quad \boldsymbol{L}_{f r}=\boldsymbol{L}_{r f}^{T},
$$

where $\theta_{r}$ is rotor angular position.

In order to reduce the number of model variables, Concordia transformation is employed to convert from three phase quantities $a b c$ to two phase quantities $\alpha \beta$ coordinate. The transformation is defined as,

$$
\left[\begin{array}{l}
u_{\alpha} \\
u_{\beta}
\end{array}\right]=\sqrt{\frac{2}{3}}\left[\begin{array}{cc}
1-1 / 2 & -1 / 2 \\
0 \sqrt{3} / 2 & -\sqrt{3} / 2
\end{array}\right]\left[\begin{array}{l}
u_{a} \\
u_{b} \\
u_{c}
\end{array}\right] .
$$

\section{Stator short circuit fault diagnosis using parameter estimation}

The mathematical model indicates that a stator short circuit fault can be described by the fault level $\eta_{f}$ and fault localization $\theta_{f}$. Therefore, it is feasible to detect and locate a stator short circuit fault by using the parameter estimation method to identify $\eta_{f}$ and $\theta_{f}$. The parameter estimation procedure is sketched in Fig. 1 . The proposed condition monitoring of stator windings scheme is conducted using the SIMULINK induction motor model, developed in [23]. The model implementation details in the SIMULINK environment will not be discussed here. In the proposed method, the parameter values from parameter estimation algorithm are imported to the machine model. Based on these parameters, the stator currents $i_{\alpha}^{\prime}$ and $i_{\beta}^{\prime}$ are generated. The generated currents $i_{\alpha}^{\prime}$ and $i_{\beta}^{\prime}$ are compared with measured $i_{\alpha}$ and $i_{\beta}$, which are converted from recorded currents $i_{a, b, c}$ in $a b c$ coordinates using Eq. 10. The difference between simulated and measured currents, i.e. the cost function, is 


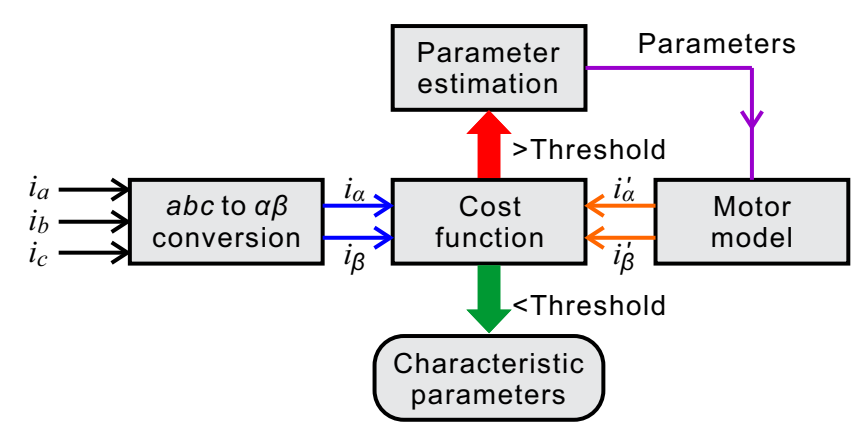

Figure 1. Induction motor stator winding short circuit fault detection using parameter estimation.

defined as

$$
J=\sum_{k=1}^{N}\left\{\left[i_{\alpha}(k)-i_{\alpha}^{\prime}(k)\right]^{2}+\left[i_{\beta}(k)-i_{\beta}^{\prime}(k)\right]^{2}\right\},
$$

where $N$ is number of samples. If $J$ is bigger than a threshold, the selected parameter estimation method will generate a new set of parameters and transfer them to the induction motor model. The process iterates until the threshold is met. The stator short circuit fault can be revealed by the estimated characteristic parameters $\eta_{f}$ and $\theta_{f}$. It is worthwhile to notice that the proposed parameter estimation method can be adapted to monitor and diagnose other faults in different types of motors by simply changing the corresponding motor model and characteristic parameters.

The selection of parameter estimation algorithm is critical since the induction motor model is nonlinear and the cost function might include several local minima. In this study, the PSA and GA are employed to illustrate the proposed induction motor fault diagnosis method. The PSA is the direct local search methods, which does not need information on either gradient or Hessian of the objective function [19]. The PSA finds minimum by generating a sequence of points approaching an optimal point. As the search progresses, the values of the objective function for generated sequence of points will either decrease or keep constant. Considering a 
$D$-dimensional objection function $f(X)$, the algorithm starts from an initial point $X_{0}=\left[x_{1}, x_{2}, \ldots, x_{D}\right]$ and initial mesh of $M_{0}$. The value of $M_{0}$ should be a positive scalar. In this study, the initial mesh size $M_{0}=1$ is utilized. Sequence of search pattern vectors are generated by either $2 D$ or $D P 1$ methods [19]. In the case of $2 D$ method, $2 \times D$ new vectors are generated, i.e.

$$
\left[\begin{array}{c}
x_{1} \\
x_{2} \\
\cdots \\
x_{D}
\end{array}\right] \pm(\underbrace{\left[\begin{array}{c}
1 \\
0 \\
\ldots \\
0
\end{array}\right],\left[\begin{array}{c}
0 \\
1 \\
\ldots \\
0
\end{array}\right],\left[\begin{array}{c}
0 \\
0 \\
\ldots \\
1
\end{array}\right]}_{D}) \times M_{0}
$$

Only $D+1$ new vectors are generated in the $D P 1$ method, i.e.

$$
\left[\begin{array}{c}
x_{1} \\
x_{2} \\
\ldots \\
x_{D}
\end{array}\right]+(\underbrace{\left[\begin{array}{c}
1 \\
0 \\
\ldots \\
0
\end{array}\right],\left[\begin{array}{c}
0 \\
1 \\
\ldots \\
0
\end{array}\right],\left[\begin{array}{c}
0 \\
0 \\
\ldots \\
1
\end{array}\right]}_{D},\left[\begin{array}{c}
-1 \\
-1 \\
\ldots \\
-1
\end{array}\right]) \times M_{0}
$$

If the smallest function value $f\left(X_{i}\right)$ obtained via local search based on pattern vectors is smaller than $f\left(X_{0}\right)$ (called a successful poll), the $X_{i}$ is selected as central point $X_{c}$, which is used to generate new pattern vectors with the mesh size of $M_{c} \times F_{E}$, where $M_{c}$ is the current mesh size and $F_{E} \geq 1$ is mesh expansion factor. $F_{E}$ specifies the mesh size after a successful poll. In this study, the $F_{E}$ is set to 2, which means that the size of the mesh is multiplied by 2 after a successful poll. If the function values of all new generated vectors are bigger than $f\left(X_{0}\right)$ (called an unsuccessful poll), the central point $X_{c}$ remains the same and the mesh size is reduced to $M_{c} / F_{C}$, where and $F_{C} \geq 1$ is mesh contraction factor. The $F_{C}$ is also set to 2 , which means that the size of the mesh is divided by 2 after an unsuccessful poll. The algorithm iterates until the stopping criterion is fulfilled. In our algorithm, the stopping criterion is set to FunTol, i.e. the difference between the function values of the previous and current central point is less than FunTol. As a direct search 
method, the parameter estimation result of the PSA depends on the starting point. This is analyzed and discussed in the Section 4.

The GA is considered as a global optimization method and it is independent of starting point [20]. The algorithm is inspired by natural evolution, such as inheritance, mutation, selection, and crossover. The GA starts with creating a random initial population. The algorithm then breed a new generation based on the existing population. The selected members, called parents, are chosen based on their fitness. Some members with lower fitness are directly passed to the next population, named elite. Children are generated either by mutation (random choosing a sing parent) or crossover (combining the vector entries of a pair of parents). Then, the current population is replaced by the new generated children. The process continues until one of the stopping criteria is met. For the fair comparison, the stopping criterion of the PSA and GA is set the same value of FunTol.

\section{Experimental Results and Discussion}

The parameter estimation case study is devised by using a three phase $800 \mathrm{~W}$ induction motor with constant voltage and frequency power supply. The parameters of the motor are tabulated in Table. 1. Fig. 2 presents a schematic diagram of the experiment setup. Each phase of the stator winding contains 4 coils. Each coil consists of 90 turns made of enameled wire with a diameter of $0.6 \mathrm{~mm}$. In order to make stator short circuit fault, the insulation layer of enameled wire of one coil in Phase $b$ is scraped and soldered with wires. These wires are connected to a switch box which controls the stator short-circuit fault level. For example, $10 \%$ winding short circuit fault means that 9 coil turns are shortened through the switch box. The rotor shaft of the induction motor is attached to a DC generator as a loading. 


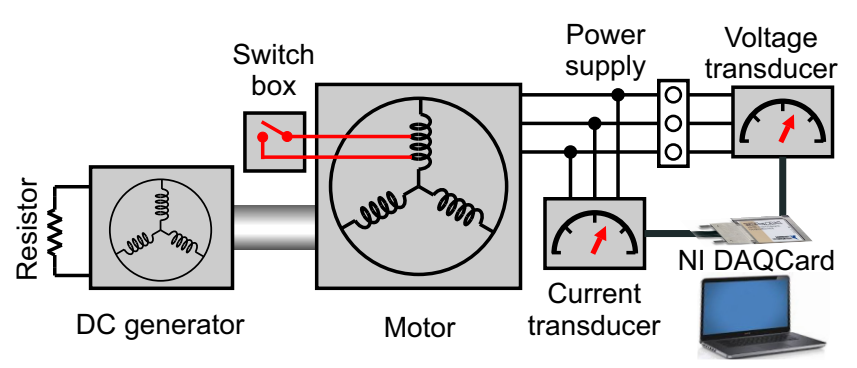

Figure 2. Induction motor stator winding short circuit fault detection experimental setup.

Table 1

Induction motor parameters

\begin{tabular}{ll}
\hline Ouput power & $800 \mathrm{~W}$ \\
Line voltage & $380 \mathrm{~V}$ \\
Rotor inertia & $0.0025 \mathrm{Kg} \mathrm{m}$ \\
Rated frequency & $50 \mathrm{~Hz}$ \\
Rated current & $2.2 \mathrm{~A}$ \\
No load speed & $1497 \mathrm{rpm}$ \\
Number of poles & 4 \\
Rated power factor & 0.74 \\
Stator winding resistance & $8.4 \mathrm{ohm}$ \\
Stator winding reactance & $10.3 \mathrm{ohm}$ \\
Stator magnetizing reactance & $137.5 \mathrm{ohm}$ \\
Referred rotor winding resistance & $8.2 \mathrm{ohm}$ \\
Referred rotor winding reactance & $10.3 \mathrm{ohm}$ \\
\hline
\end{tabular}

The output of the DC generator is connected to a resistor. The loading level is adjusted by a gear box. The loading level $L$ is also estimated by the algorithm since it is an important factor for motor condition monitoring and control. The stator voltage and current of the induction motor are measured by the voltage transducer LEM LV 25-P [24] and the current transducer LEM HY-15 [25], respectively. The signals from these two transducers are recorded by the National Instruments (NI) 16-bit DAQCard-6036E PCMCIA card [26]. The sampling frequency is $10 \mathrm{kHz}$.

The stator current signals $i_{\alpha}^{\prime}$ and $i_{\beta}^{\prime}$ are generated from the induction motor model based on the measured voltage signals. The generated current signals are syn- 
Table 2

Boundaries of under estimated parameters

\begin{tabular}{|c|c|c|}
\hline$\eta_{f}$ & Fault level & {$\left[\begin{array}{ll}0 & 0.3\end{array}\right]$} \\
\hline$\theta_{f}$ & Fault localization & $\{0,1,2\}$ \\
\hline$L$ & Loading level & {$\left[\begin{array}{ll}0 & 1\end{array}\right]$} \\
\hline$T_{s}$ & Offset time & {$\left[\begin{array}{ll}0 & 0.02\end{array}\right]$} \\
\hline
\end{tabular}
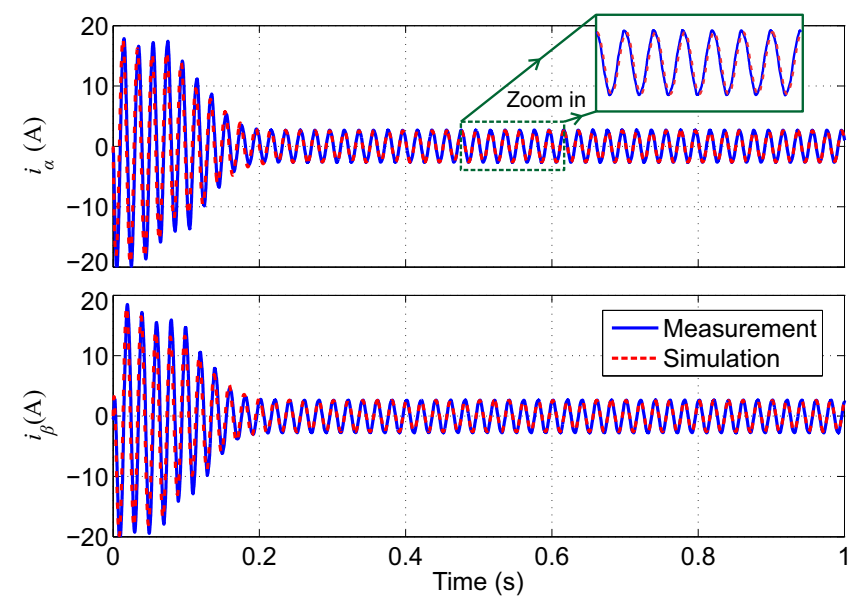

Figure 3. The unsynchronized simulated and measured current singles due to the measurement error. A parameter $T_{s}$ is defined to synchronize simulated and measured current singles.

chronized with the measured voltage signals. The unsynchronized measured voltage and current signals, which can result from measurement error, leads to the time offset between simulated and measured current signals, as shown in Fig 3. Therefore, an additional parameter $T_{s}$ is defined to synchronize simulated and measured currents. All realistic ranges where parameter variables can vary are listed in Table. 2. The range of fault level $\eta_{f}$ is from 0 to 0.3 , i.e. from healthy condition to $30 \%$ stator short circuit fault in one coil. The loading level $L$ is defined in the range from 0 to 1 (100\% loading level). Since the rated frequency of the supply voltage is $50 \mathrm{~Hz}$, the offset time is adjusted within one period of $0.02 \mathrm{~s}$.

The experiment data of $10 \%\left(\eta_{f}=0.1\right)$ stator short circuit fault in Phase $b$ $\left(\theta_{f}=1 \times 2 \pi / 3\right)$ is employed to evaluate the proposed diagnostic technique based 
on parameter estimation. The motor is under $50 \%$ loading. The data windows is from 0.5 to $1 \mathrm{~s}$, i.e. motor reaches steady state, as shown in Fig. 3. With the sample frequency of $10 \mathrm{kHz}$, the total data points $N$ is equal to 5001 for both $i_{\alpha}$ and $i_{\beta}$ in the cost function Eq.11. In the first attempt, the staring point of the PSA is set to the left boundary of each parameter. The key parameters of PSA are discussed as follows. The parameter CompletePoll is set to "on", which means the algorithm evaluates all points in the poll. If the parameter is set to "off", the algorithm stops once the function value of a evaluated point is smaller than that of the central point, which will increase the risk of being trapped into local minima. Since the running SIMULINK induction motor model takes longer time than the algorithm itself, the points that the algorithm have already visited are stored to in the cache to avoid being calculated again. So, the parameter Cache is set to "on". Figs. 4(a)-(d) and (e) show the trajectories of estimated parameters and cost function value, respectively. The algorithm reaches the stopping criterion of $F u n T o l=10^{-5}$, i.e. the difference between the function values of the previous and current central point is less than $10^{-5}$. The estimated parameters are $\left[\eta_{f}=0 ; \theta_{f}=0 ; L=0.5300 ; T_{s}=0.0001\right]$ and the cost function value is $J=121.7344$, which is far from the minimum value.

As mentioned in the previous section, the search result of PSA depends on the starting point and it has been trapped into local minima. Thus, LHS method is utilized to generate a starting point for PSA. The LHS is a method of sampling that can generate points with each component close to evenly spaced [22]. The point with the minimal value is set to the start point of PSA. Although the extra time has been spent on evaluating points generated by the LHS method, it can reduce the risk of being trapped into local minima. The large number of sampling points 


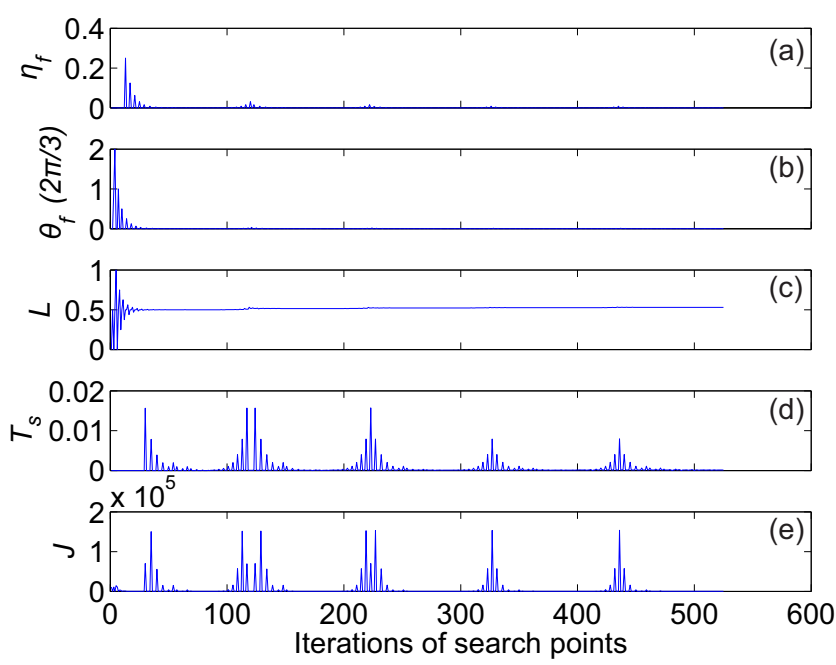

Figure 4. Trajectories of estimated parameters and cost function value using the PSA with left boundary of each parameter as a starting point.

will reduce the risk and of course increase the evaluation time. This is a trade off between accuracy and time consumption.

We use this 4 parameters estimation task as an example to illustrate the LHS method. In order to generate 5 sampling points $S P=5$ points, a matrix $M_{1}$ of 5 rows and 4 columns is firstly built by combining 4 column vectors. Each column vector has a random permutation of $[1,2,3,4,5]$. An example of matrix $M_{1}$ is shown below

$$
M_{1}=\left[\begin{array}{llll}
2 & 5 & 4 & 2 \\
1 & 3 & 1 & 1 \\
3 & 2 & 5 & 4 \\
5 & 4 & 2 & 3 \\
4 & 1 & 3 & 5
\end{array}\right]
$$

Then, the sampling points matrix $M_{L H S}$ is calculated by using the equation $M_{L H S}=\left(M_{1}-M_{r}\right) / N$, where $M_{r}$ is a random matrix with the same size as $M_{1}$. The elements of $M_{r}$ are in the range of $[0,1]$. Each element of $M_{L H S}$ is also in the range of $[0,1]$. The five rows represent 5 new generated sampling points. Finally, these points are rescaled to fit boundaries in Table. 2. 


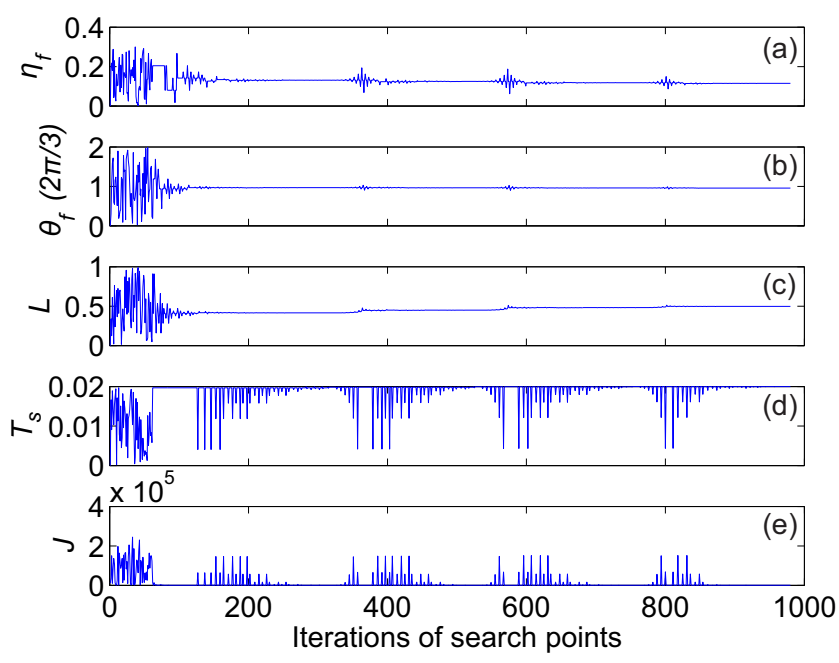

Figure 5. Trajectories of estimated parameters and cost function value using the PSA with the aid of LHS to generate a starting point.

In this parameter estimation, the total sampling points $S P$ is 60 . Within these 60 points, the point with the minimal function value is selected as the starting point of PSA. Figs. 5(a)-(d) and (e) show the trajectories of estimated parameters and cost function value, respectively. After the evaluation the 60 points generated by the LHS method, the point of $\left[\eta_{f}=0.2045 ; \theta_{f}=0.1873 \times 2 \pi / 3 ; L=0.4086 ; T_{s}=\right.$ 0.0197] is selected as the starting point of the PSA. The estimated parameters after the PSA terminated are $\left[\eta_{f}=0.1046 ; \theta_{f}=0.9569 \times 2 \pi / 3 ; L=0.4966 ; T_{s}=0.0020\right]$, which is close to the experiment setup of $10 \%$ stator circuit fault in Phase $b$. The total 980 points are evaluated with the minimal value of the cost function $J=$ 66.5986. Since points in the sampling points matrix are randomly generated by LHS method, the selected starting point might be close to local minima in a very rare occasion. Therefore, there is still a risk of the PSA being trapped into local minima. The risk can be reduced by increasing the number of sampling points $S P$. In a continuing induction motor monitoring system, the previous parameter estimation 
result can be used as a starting point for the next search. In this case, PSA can be directly applied in this system to shorten the parameter estimation time.

The GA is employed to estimate these parameters from the same recorded data. The general rules to select the key parameters of the GA are discussed as follows. The population size of each generation should be larger than number of to be estimated parameters. The large number of population size enables more evaluated points and thereby obtain a more accurate result. However, large number of population size requires long computational time. As a rule of thumb, population size is usually smaller than four times the number of searched variables to avoid long running time [20]. In this study, the population size of 20 is utilized to have an accurate result while maintain a reasonable computational time. The crossover fraction $(C F)$ is in the range of 0 and 1 , which controls that how many percent children are crossover children. The parameter of elite count $(E C)$ is an integer which specifies how many individuals in the current generation are guaranteed to survive to the next generation. These two parameters are important to control the standard deviation (SD) of each new generation. They are selected based on the study of mean and SD of the cost function values $J$ in the $10^{\text {th }}$ generation, as shown in Fig. 6. As one can observe from the figure, the small $C F$ usually leads to large SD. The small values of $C F$ and $E C$ most likely transform GA into an random search. With the increase of $E C$, the curve of the mean values of cost function becomes more flat and the SDs become smaller under various $C F$ values. The parameters of $C F=0.8$ and $E C=10$ yield the smallest mean and $\mathrm{SD}$ of the cost function value. Therefore, these two parameters values are utilized in the following study. For the fair comparison, the stopping criterion of FunTol $=10^{-5}$ is the same as that for the PSA. 


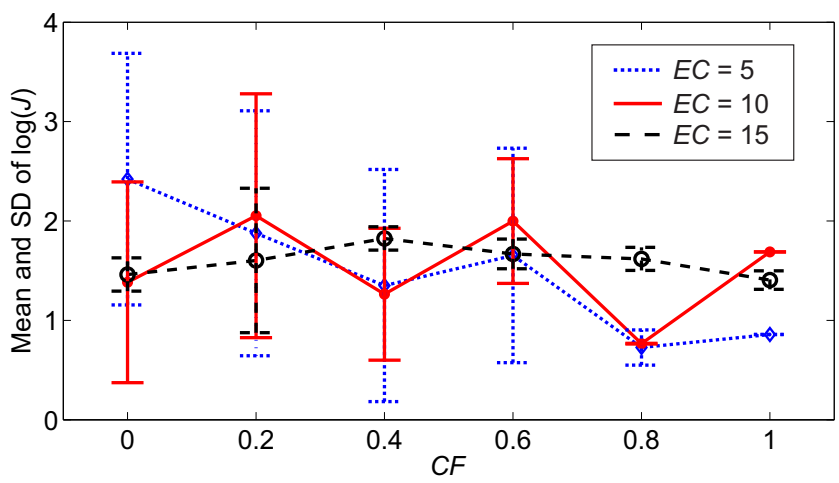

Figure 6. The means and SDs of the cost function $(J)$ values of the $10^{\text {th }}$ generation with different values of crossover fraction $(C F)$ and elite count $(E C)$.

Figs. 7(a)-(d) and (e) show the trajectories of estimated parameters and cost function value, respectively. The total number of evaluated points is 1520 . The more accurate result is obtained at the cost of increased number of evaluated points. The estimated parameters of $\left[\eta_{f}=0.1032 ; \theta_{f}=0.9798 \times 2 \pi / 3 ; L=0.5042 ; T_{s}=0.0002\right]$ correctly indicate the motor state. The minimal value of the cost function is 52.4335 , which is smaller than that of PSA. The programs were implemented on a computer with Intel i7-3770 processor (4 cores, $3.1 \mathrm{GHz}$ ) and 8 GB DRAM. The running times

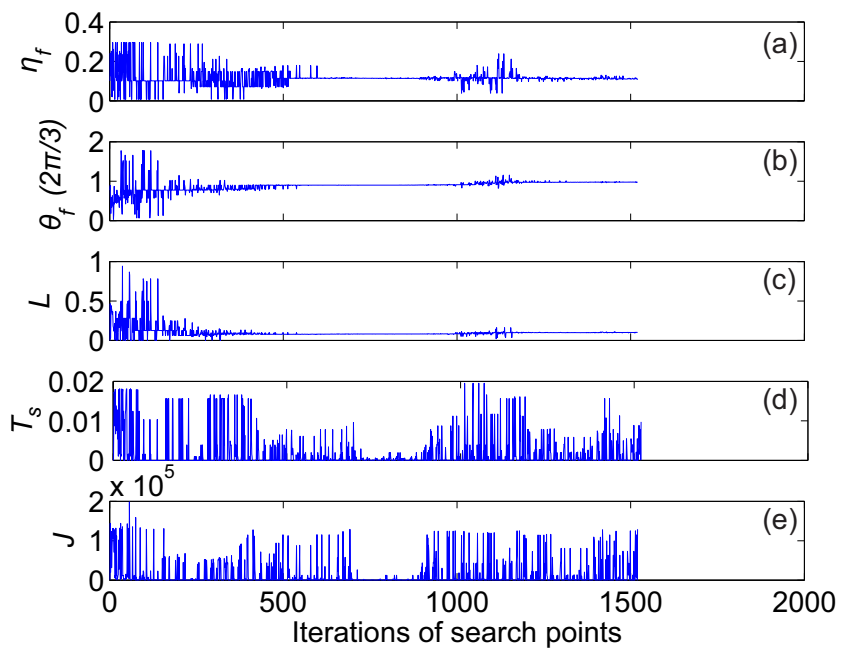

Figure 7. Trajectories of estimated parameters and cost function value using the GA. 
of the PSA and GA condition monitoring procedure are $235 \mathrm{~s}$ and $342 \mathrm{~s}$, respectively. For both algorithms, the time for evaluating one point is about $0.23 \mathrm{~s}$ and the SIMLINK model consumes about $90 \%$ of that time. Hence, the total ruining times can be reduced by simplifying mathematical model as suggested in [27]. Although the GA requires more computational time compared to the PSA (i.e. more evaluated points), it guarantees robustness in parameter estimation.

The proposed method has been verified using the same three phase $800 \mathrm{~W}$ induction motor under different combination of loading levels and inter-turn stator short circuit fault levels in Phase b. Fig. 8 shows the comparison between exper-
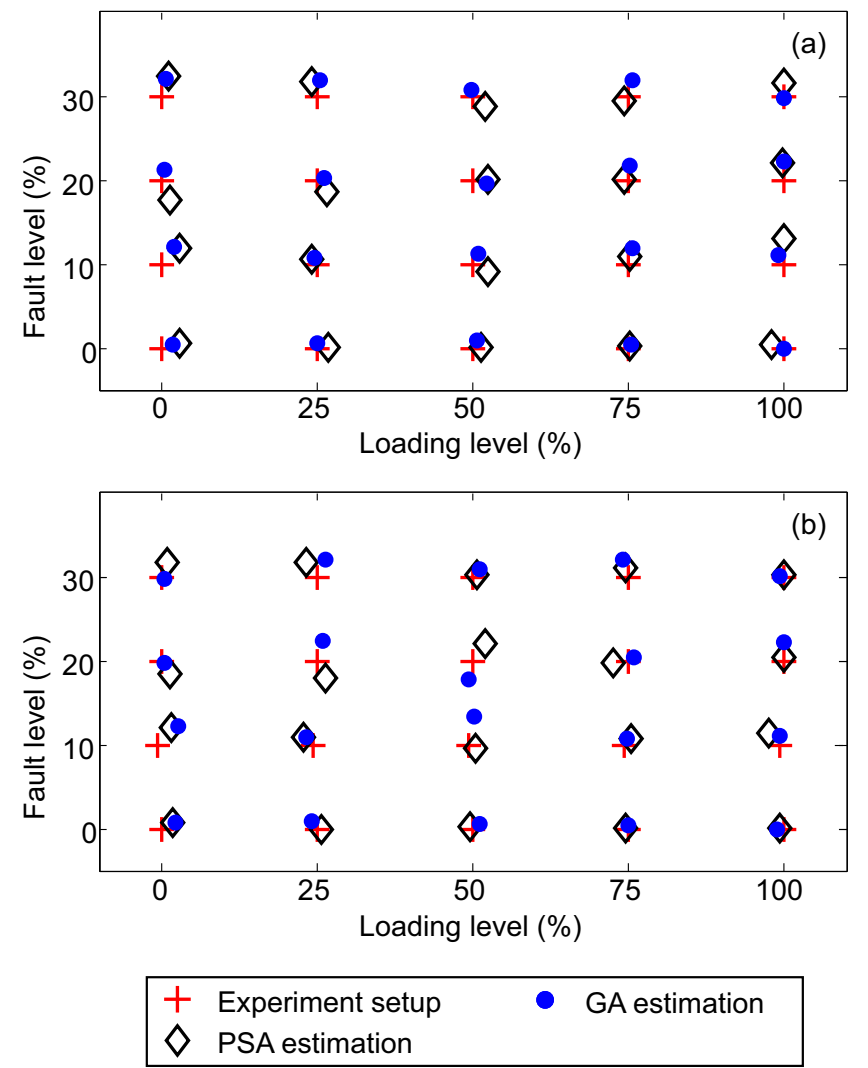

Figure 8. Comparison between experiment setup with parameter estimated results by using PSA and GA. The stopping criterion of (a). FunTol $=10^{-5}$ and (b). maximum cost function evaluations of 1000, respectively. 
iment setup with estimated results by using PSA and GA. For both algorithms, the results in Figs. 8(a) and (b) are obtained by using the stopping criterion of FunTol $=10^{-5}$ and maximum cost function evaluations of 1000, respectively. In all parameter estimations, the fault location is successfully detected in Phase $b$ and is not shown in the figure. The parameter $T_{s}$ is not plotted since it is only utilized to calibrate the time offset. The red crosses represent the experiment setup. The estimated results of parameters $\eta_{f}$ and $\theta_{f}$ by using PSA and GA are indicated with black diamonds and blue dots, respectively. The estimated parameters $\eta_{f}$ and $\theta_{f}$ have clearly indicated the motor state. Given the stopping criterion of FunTol $=10^{-5}$, in most cases, the estimated results from GA is closer to the experiment setup than these from PSA, as shown in Fig. 8(a). The evaluated points of PSA are around 1000, while GA evaluates about 500 points more than PSA under these combinations of loading and fault levels. In Fig. 8(b), the stopping criterion is set to the maximum cost function evaluations of 1000 , i.e. the both algorithms stop after evaluating 1000 points. The similar results are obtained by using PSA due to the approximately equal number of function evaluations as in Fig. 8(a). With the limited number of function evaluations, the large value of $E C$ or $C F$ has to be utilized to decrease the diversity of subsequent generations of GA. However, large value of $E C$ or $C F$ might increase the value of cost function and hence the error of estimated parameters, as shown in Fig. 6. In Fig. 8(b), the estimated parameters from GA are obtained with the $E C=10$ and $C F=1$. In this case, the related errors of estimated parameters from GA are larger than these in Fig. 8(a). The estimated results from GA are still comparable with these from PSA. 


\section{Stator short circuit faults diagnosis under variable motor pa- rameters and unbalanced voltage supply}

Motor parameters might drift from the values given in the data sheet due to the environment factors (e.g. temperature and humidity) and working condition (e.g. load level and alignment). In order to verify the impact of variable motor parameters on the proposed method, the parameters of the motor are changed in the model to generate stator current. Then, this stator current is utilized to estimate stator short winding faults. During the fault diagnosis process, the model uses the original values of motor parameters. Since stator winding resistance and reactance are most likely to be affected during motor operation, these two parameters are chosen to examine the proposed method under the low fault level of $3 \%$ inter-turn stator winding circuit fault in Phase $b$. Motor load level is set to $10 \%$. The stopping criterion of PSA and GA is set to maximum 400 evaluated points. Figs. 9(a)-(c) show that the deviations of the estimated parameters based on PSA linearly rise with the increase of resistance and reactance offset. The change of stator winding resistance has more effect on the load level estimation. Figs. 9(d)-(f) show the diagnosis results of the combination of stator winding resistance and reactance offset. The results do not have significant difference compared to a single parameter offset in Figs. 9(a)-(c). In both cases, the fault can be successfully located in Phase $b$. The faults diagnosis results based on GA are shown in Fig. 10. The similar results can be observed that stator winding resistance offset has more effect on load level estimation. The combination of offsets in two parameters does not decrease the performance of GA. The deviations of estimated parameters from GA are larger than that from 

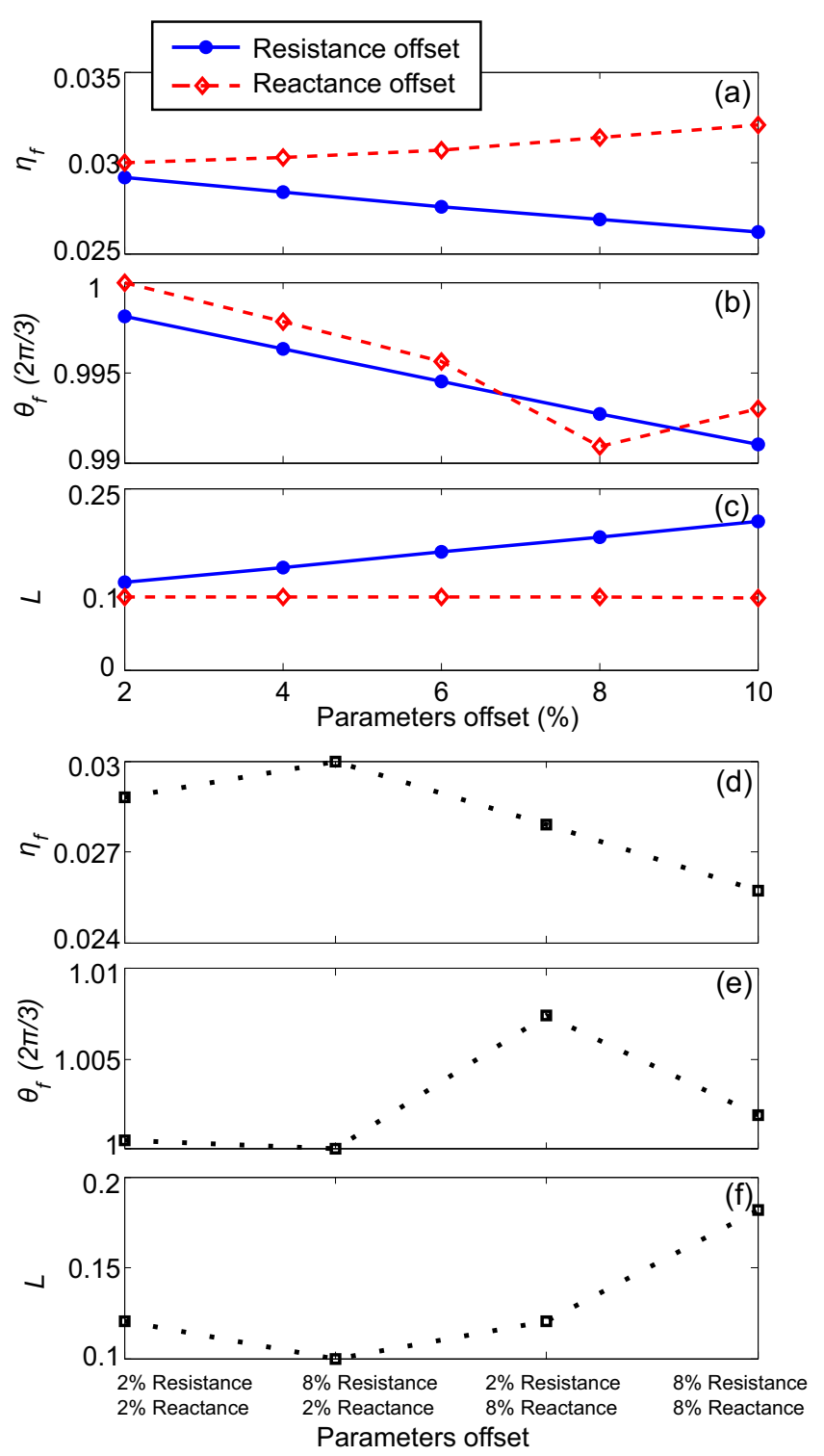

Figure 9. Stator winding short circuit faults diagnosis under variable stator winding resistance and reactance using PSA. (a)-(c): Estimated parameters with stator winding resistance and reactance offset; (d)-(f): Estimated parameters with the combination of stator winding resistance and reactance offset.

PSA. Given the limited computational time (i.e. evaluated points), PSA has better performance than GA in term of the accuracy of estimated parameters.

Unbalanced voltage is a common disturbance in electrical systems. The unbalanced voltage has negative effects on both motor operation and fault diagnosis. The 

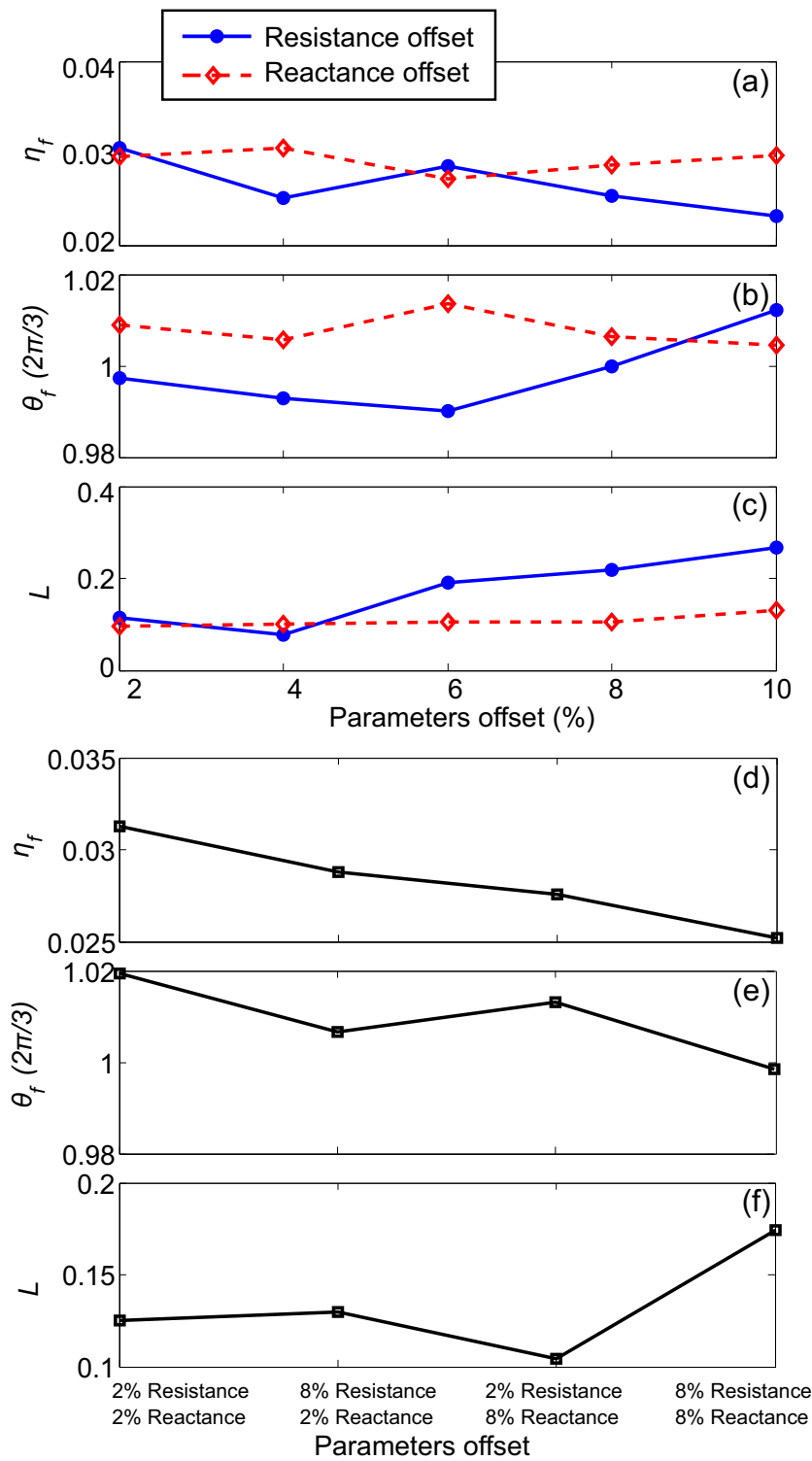

Figure 10. Stator winding short circuit faults diagnosis under variable stator winding resistance and reactance using GA. (a)-(c): Estimated parameters with stator winding resistance and reactance offset; (d)-(f): Estimated parameters with the combination of stator winding resistance and reactance offset.

voltage unbalance factor (VUF) is defined as the ratio of positive $\left(V_{p}\right)$ and negative $\left(V_{n}\right)$ sequence voltage components [28]

$$
V U F=\frac{V_{p}}{V_{n}} \times 100 \%,
$$




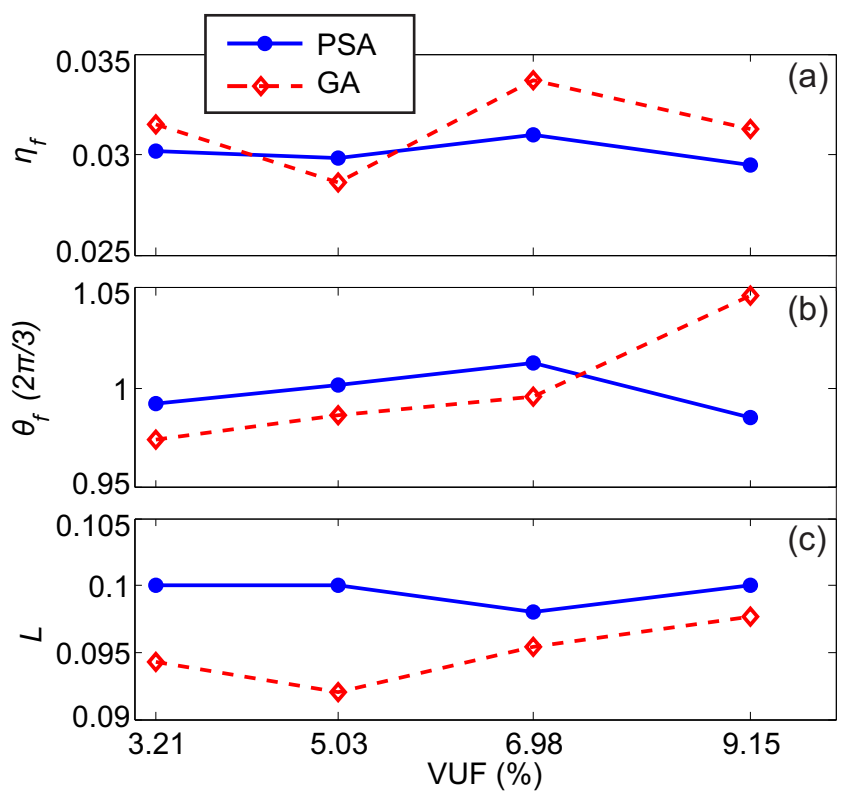

Figure 11. Stator winding short circuit faults diagnosis under unbalanced voltage supply.

These two components are given by

$$
\begin{aligned}
& V_{p}=\frac{V_{a}+\alpha_{1} \cdot V_{b}+\alpha_{2} \cdot V_{c}}{3}, \\
& V_{n}=\frac{V_{a}+\alpha_{2} \cdot V_{b}+\alpha_{1} \cdot V_{c}}{3},
\end{aligned}
$$

where $\alpha_{1}=1 \angle 120^{\circ}$ and $\alpha_{2}=1 \angle 220^{\circ}$. Fig. 11 shows the estimated results using PSA and GA by considering both magnitude and phase unbalance. For example, the $V U F=3.21 \%$ is obtained by setting three phase voltages of $V_{a}=363 \cos (\omega t)$, $V_{b}=279 \cos \left(\omega t+\angle 280^{\circ}\right)$ and $V_{c}=335 \cos \left(\omega t+\angle 122^{\circ}\right)$, where $\omega$ is base electrical radians per second. The motor setting and algorithm parameters are the same as the previous study. Fig. 11 shows that both fault level, fault location and load level can be detected by using PSA and GA algorithm over the range of VUF up to $9.15 \%$. 


\section{Conclusion}

Induction machine stator fault diagnosis is achieved by estimating the selected set of the machine model parameters using voltage and current signals recorded at machine power supply terminal. The PSA and GA are applied in the parameter estimation method. The experimental results reveal that the accuracy of direct local search method (PSA) highly depends on the algorithm initial point in the parameter space. The risk of being trapped into local minima has been significantly reduced by introducing the LHS method. The PSA is suitable for continuous monitoring system where the new search starts from the previous result. The fault diagnostic based on the GA is not sensitive to initial search point and achieves accurate estimation results and guaranteed detection of global minima with increased computation time. The proposed model-based parameter estimation technique is robust to the motor parameters offset and unbalanced voltage supply. As a generic technique, the method can be utilized to diagnose other types of machines by using the corresponding machine models.

\section{References}

[1] P. Vas, Parameter Estimation, Condition Monitoring, and Diagnosis of Electrical Machines. $\quad$ Clarendron Press Oxfords, 1993, ch. 3.

[2] D. Dorrell, W. Thomson, and S. Roach, Analysis of airgap flux, current, and vibration signals as a function of the combination of static and dynamic airgap eccentricity in 3-phase induction motors, IEEE Trans. Ind. Appl., Vol. 33, No. 1, Jan. 1997, pp. $24-34$.

[3] K. Gyftakis, D. Spyropoulos, J. Kappatou, and E. Mitronikas, A novel approach for broken bar fault diagnosis in induction motors through torque monitoring, IEEE 
Trans. Energy Convers., Vol. 28, No. 2, 2013, pp. 267-277.

[4] A. Sadoughi, M. Ebrahimi, and E. Razaei, A new approach for induction motor broken bar diagnosis by using vibration spectrum, in International Joint Conference SICE-ICASE, 2006., Oct. 2006, pp. $4715-4720$.

[5] J.-H. Jung, L. Jong-Jae, and B.-H. Kwon, Online diagnosis of induction motors using MCSA, IEEE Trans. Ind. Electron., Vol. 53, No. 6, 2006, pp. 1842-1852.

[6] S. M. A. Cruz and A. J. M. Cardoso, Stator winding fault diagnosis in three-phase synchronous and asynchronous motors, by the extended Park's vector approach, IEEE Trans. Ind. Appl., Vol. 37, No. 5, 2001, pp. 1227-1233.

[7] A. da Silva, R. Povinelli, and N. A. O. Demerdash, Induction machine broken bar and stator short-circuit fault diagnostics based on three-phase stator current envelopes, IEEE Trans. Ind. Electron., Vol. 55, No. 3, 2008, pp. 1310-1318.

[8] M. Bouzid, G. Champenois, N. Bellaaj, L. Signac, and K. Jelassi, An effective neural approach for the automatic location of stator interturn faults in induction motor, IEEE Trans. Ind. Electron., Vol. 55, No. 12, 2008, pp. 4277-4289.

[9] S. Nandi, Detection of stator faults in induction machines using residual saturation harmonics, IEEE Trans. Ind. Appl., Vol. 42, No. 5, 2006, pp. 1201-1208.

[10] C. De Angelo, G. Bossio, S. Giaccone, M. Valla, J. Solsona, and G. Garcia, Online model-based stator-fault detection and identification in induction motors, IEEE Trans. Ind. Electron., Vol. 56, No. 11, 2009, pp. 4671-4680.

[11] M. Ben Khader Bouzid and G. Champenois, New expressions of symmetrical components of the induction motor under stator faults, IEEE Trans. Ind. Electron., Vol. 60, No. 9, 2013, pp. 4093-4102.

[12] G. Joksimovic, J. Riger, T. Wolbank, N. Peric, and M. Vasak, Stator-current spectrum signature of healthy cage rotor induction machines, IEEE Trans. Ind. Electron., Vol. 60, No. 9, 2013, pp. 4025-4033. 
[13] A. Bellini, A. Yazidi, F. Filippetti, C. Rossi, and G.-A. Capolino, High frequency resolution techniques for rotor fault detection of induction machines, IEEE Trans. Ind. Electron., Vol. 55, No. 12, 2008, pp. 4200-4209.

[14] A. Bouzida, O. Touhami, R. Ibtiouen, A. Belouchrani, M. Fadel, and A. Rezzoug, Fault diagnosis in industrial induction machines through discrete wavelet transform, IEEE Trans. Ind. Electron., Vol. 58, No. 9, 2011, pp. 4385-4395.

[15] M. Pineda-Sanchez, R. Puche-Panadero, M. Riera-Guasp, J. Perez-Cruz, J. RogerFolch, J. Pons-Llinares, V. Climente-Alarcon, and J. Antonino-Daviu, Application of the teager-kaiser energy operator to the fault diagnosis of induction motors, IEEE Trans. Energy Convers., Vol. 28, No. 4, 2013, pp. 1036-1044.

[16] B. Xu, L. Sun, L. Xu, and G. Xu, Improvement of the hilbert method via esprit for detecting rotor fault in induction motors at low slip, IEEE Trans. Energy Convers., Vol. 28, No. 1, 2013, pp. 225-233.

[17] F. Duan and R. Živanović, Diagnosis of induction machine stator faults by parameter estimation techniques based on direct search on sparse grid, in The 9th IET International Conference on Advances in Power System Control, Operation and Management, 2012, pp. 1-6.

[18] E. Novak and K. Ritter, Global optimization using hyperbolic cross points, in State of the Art in Global Optimization, C. Floudas and P. Pardalos, Eds. Kluwer Academic Publishers, 1972, pp. 19-33.

[19] M. Avriel, Nonlinear Programming: Analysis and Methods. Courier Dover Publications, 2003.

[20] M. Mitchell, An Introduction to Genetic Algorithms. MIT Press, 1999.

[21] S. Bachir, S. Tnani, J. Trigeassou, and G. Champenois, Diagnosis by parameter estimation of stator and rotor faults occurring in induction machines, IEEE Trans. Ind. Electron., Vol. 53, No. 3, 2006, pp. 963-973.

[22] R. L. Iman, Latin Hypercube Sampling. John Wiley \& Sons, Ltd, 2008. 
[23] F. Duan and R. Živanović, A model for induction motor with stator faults, in $A U$ PEC12 - 22th Australasian Universities Power Engineering Conference, Bali, Indonesia, Sep. 2012, pp. 1-4.

[24] LEM. [Online]. Available: http://www.lem.com/docs/products/lv\%2025-p.pdf

[25] LEM. [Online]. Available: http://www.lem.com/docs/products/hy_sp1_e.pdf

[26] National Instruments. [Online]. Available: http://sine.ni.com/nips/cds/view/p/lang/en/nid/11914

[27] J. Stephan, M. Bodson, and J. Chiasson, Real-time estimation of the parameters and fluxes of induction motors, IEEE Trans. Ind. Appl., Vol. 30, No. 3, May 1994, pp. $746-759$.

[28] R. Dugan, M. McGranaghan, and H. Beaty, Electrical Power Systems Quality. New York: McGraw-Hill, 1996. 